\title{
Gallstone Ileus: A Case Report and Our Clinic's Experience
}

\author{
Konstantinos Alexiou, Argyrios Ioannidis, Nikolaos Sikalias, Eleftheria Konstantinidou, \\ Athanasios Fotopoulos, Ioannis Karanikas, Nikolaos Economou \\ Department of Surgery, Sismanoglion General Hospital, Athens, Greece \\ Email: argioannid@med.uoa.gr
}

Received November 27, 2013; revised December 25, 2013; accepted January 3, 2014

Copyright (c) 2014 Konstantinos Alexiou et al. This is an open access article distributed under the Creative Commons Attribution License, which permits unrestricted use, distribution, and reproduction in any medium, provided the original work is properly cited. In accordance of the Creative Commons Attribution License all Copyrights (C) 2014 are reserved for SCIRP and the owner of the intellectual property Konstantinos Alexiou et al. All Copyright (C) 2014 are guarded by law and by SCIRP as a guardian.

\section{ABSTRACT}

We present a 72-year-old female patient complaining of pain and distention of the abdomen, nausea and vomiting. No history of previous abdominal surgery, hernias or biliary disease was present. Plain abdominal x-rays showed small bowel obstruction. This mechanical obstruction of the gastrointestinal truct was caused by a gallstone in the terminal ileum.

\section{KEYWORDS}

\section{Gallstone Ileus}

\section{Introduction}

Gallstone ileus is perhaps the most insidious and difficult to diagnose cause of mechanical intestinal obstruction. It is often misdiagnosed and carries a significant rate of morbidity and mortality.

\section{Case Report}

A 72-year-old, female, patient presented with a 36-hour history of abdominal distention, colic pain and bilious vomiting. There was no history of abdominal surgery. The patient's abdomen was distended, although soft, with increasing bowel sounds. Physical examination disclosed evidence of hernia. Concomitant diseases involved cardiovascular disorders and diabetes mellitus. Laboratory tests were nonspecific and showed median leucocytosis, dehydration and electrolyte imbalance. The patient underwent nasogastric intubations and correction of electrolyte disturbances. Plain radiographs of abdomen were compatible with partial small bowel obstruction. Contrast $\mathrm{x}$-ray films showed aerobilia and distoped radiopaque abnormality (Figure 1). To clarify the etiology, an abdominal computed tomography scan was obtained. The CT findings showed aerobilia, cholecystoduodenal fistula, dilated small bowel and an intraluminal abnormality in

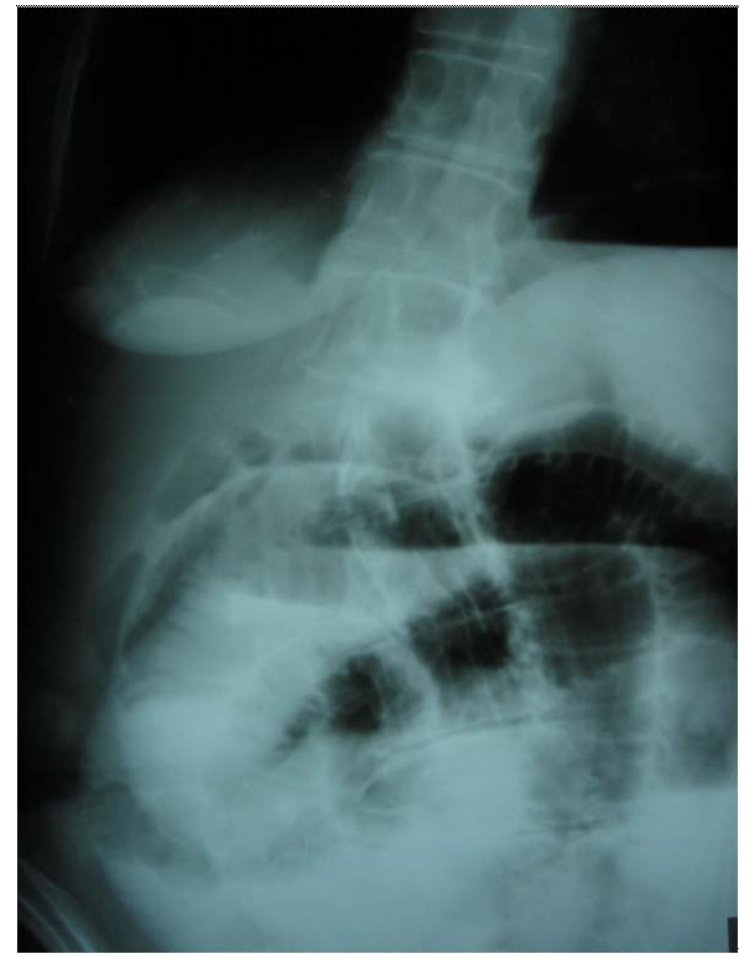

Figure 1. Contrast abdominal $x$-ray showing pneumobilia and small bowel obstruction. 
the terminal ileum (Figure 2). Duration between admission and operation was 48 hours.

Operative intervention confirmed the presence of a 3.5 cm obstructing calculus in the terminal ileum (Figure 3). Removal of the stone via enterotomy was the technique of choice and a longitudinal incision was performed, on the antimesentericic border proximal to the site of impaction, removal of the calculus and closure of the bowel wall was performed transversely, to avoid narrowing of

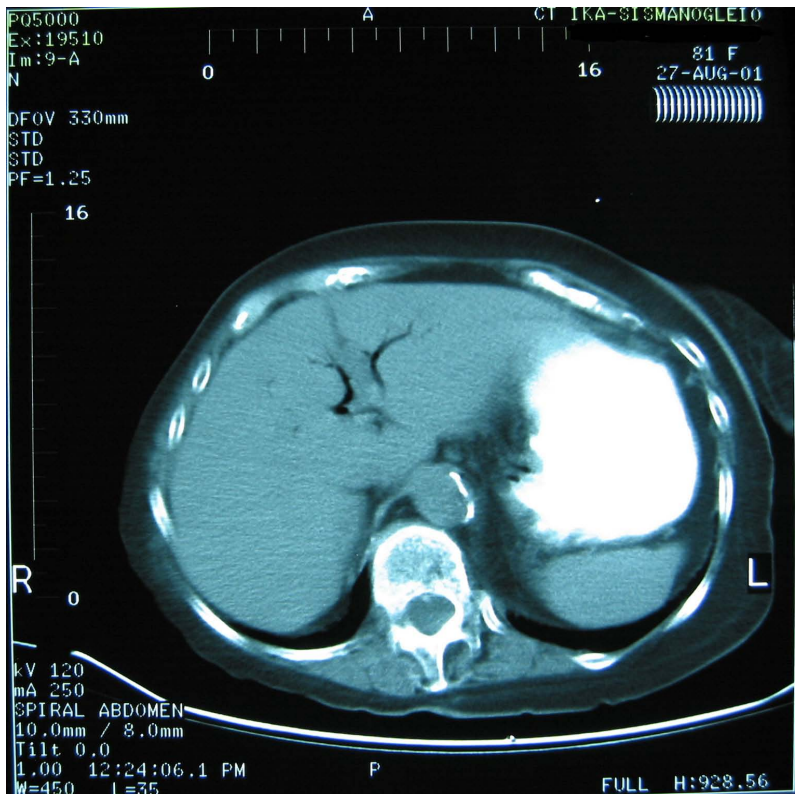

(a)

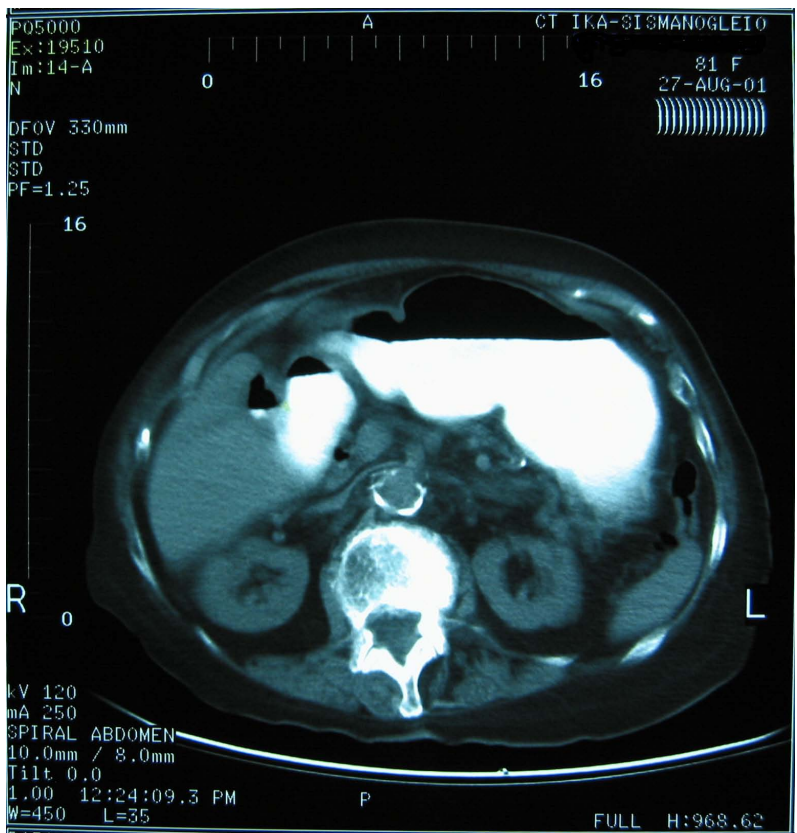

(c) the intestinal lumen. We carefully inspected the remainder of the small bowel for additional calculi. Due to the poor general condition of the patient and concomitant diseases, a cholecystectomy and cholecystoduodenal fistula closure were not carried out.

The patient recovery was successful with no postoperative complications.

Review of the hospital surgical department records from 1992-2002 revealed 7 further cases of gallstone ileus.

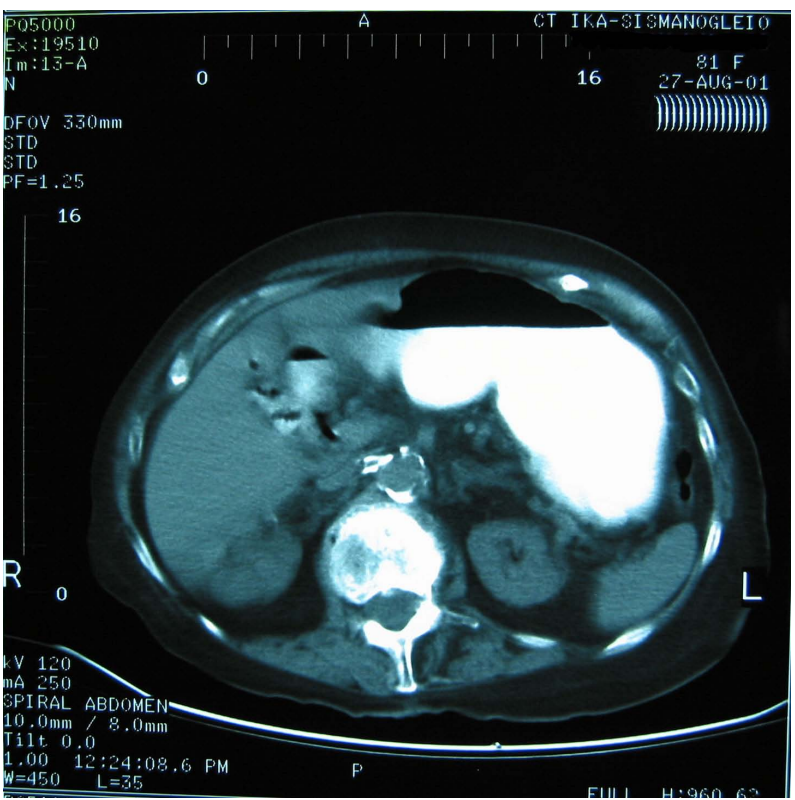

(b)

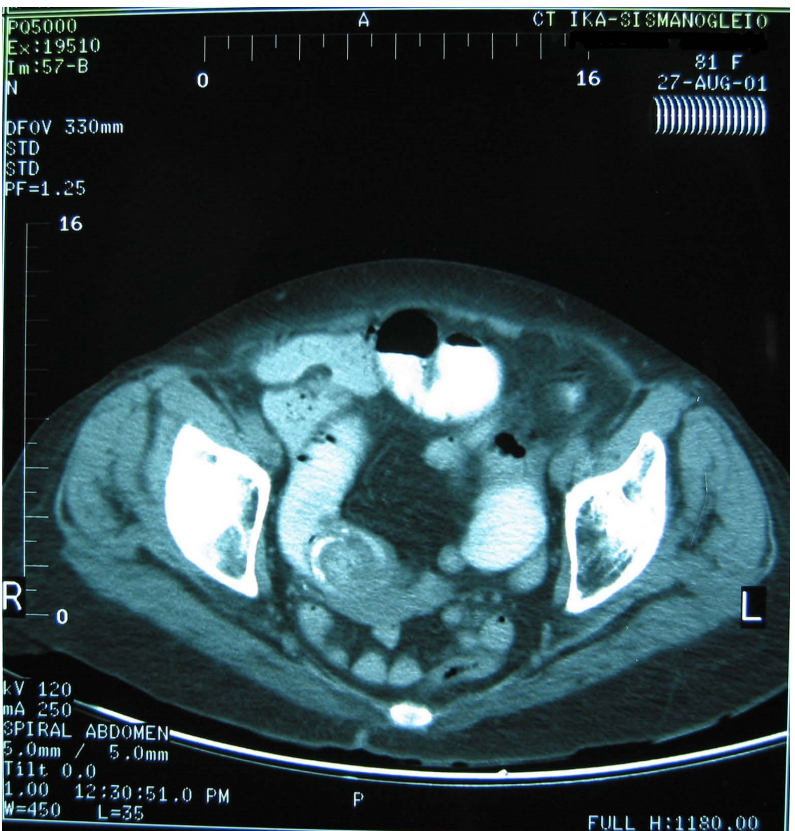

(d)

Figure 2. (a) Upper abdominal CT-scan: pneumobilia; (b) Upper abdominal CT-scan: pneumobilia; (c) Upper abdominal CTscan: cholecystoduodenal fistula; (d) Lower abdominal CT-scan of the same patient representing impacted gallstone in the terminal ileum and small bowel obstruction. 


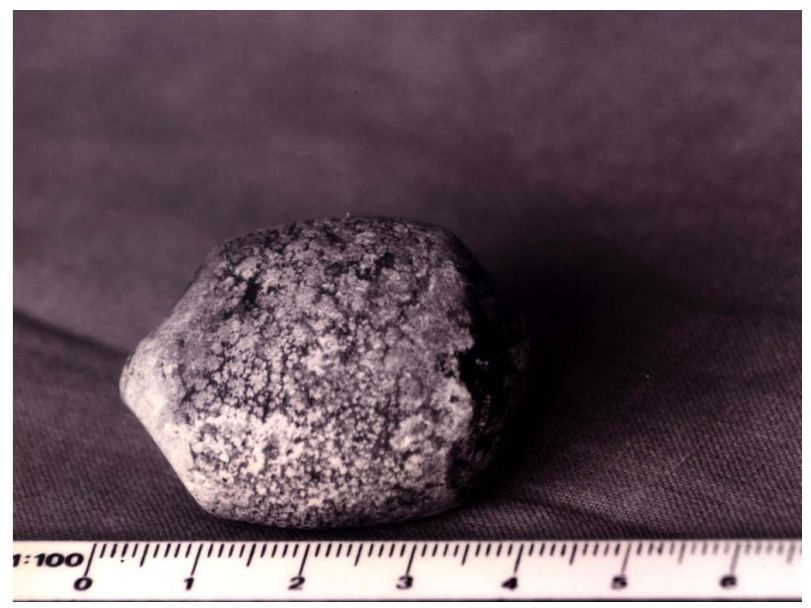

Figure 3. Extracted gallstone from small bowel.

\section{Discussion}

Gallstone ileus is a geriatric surgical emergency accounting for approximately $1 \%$ - $4 \%$ of cases of mechanical intestinal obstruction. The mortality associated with GI ranges between $12 \%$ and $27 \%$ [1]. The patients are usually older than 65 years old [2]. The disease occurs more frequently in women $[3,4]$. The median age in our series was 72 years (range 65 - 81) including seven female patients and one male.

The clinical presentation of gallstone ileus is rarely specific and more than one third of the patients have no history of biliary symptoms $[5,6]$. In our series more than half of the patients had a suspicious history consistent of biliary disorders (simple biliary colic, nausea, vomiting) and previous cholecystitis.

During the inflammation the body attempts to compartmentalize the process surrounding by soft tissues (omentum, nearby bowel including the duodenum) the diseased area. If the inflammatory process is sufficient in intensity and duration, then the diseased gallbladder can form a fistulous communication most commonly to duodenum (cholecystoduodenal fistula), but can also involve the colon, stomach, more distal segments of small intestine or the distal common bile duct [1]. In our series all the patients had cholecystoduodenal fistula.

The clinical features of gallstone ileus are similar to those of mechanical bowel obstruction $[7,8]$. The most common clinical presentation was abdominal pain and vomiting (bilious vomitus and gastric dilatation in proximal obstruction and faeculent vomitus in distal obstruction ). All the patients underwent nasogastric intubation.

Laboratory tests are usually nonspecific. In our series all the patients had leukocytosis, dehydration and electrolyte imbalance. Liver function tests were abnormal in 3 patients although these data have little diagnostic importance.

Concomitant geriatric diseases are present in as many as $80 \%$ - $90 \%$ of cases $[9,10]$. In our series 6 patients had concomitant disease, involving cardiovascular disorders, pulmonary disorders and diabetes mellitus. Four out of eight patients had a history of abdominal surgery. In 1941, Rigler et al. described the signs on plain abdominal radiography: 1) pneumobilia, 2) intestinal obstruction, 3) aberrantly located gallstone and 4) change in position of the demonstrated gallstone [11]. The first two of three sings are present in more than $40 \%$ of cases. In 1978 , Balthazar et al. described a radiological sign consisting of two adjacent small air fluid levels in the right upper quadrant, the median collection being in the duodenal bulb and the lateral in the gallbladder [12]. Contrast $\mathrm{x}$-ray films with gastrographin contrast material proved useful in detecting the course of fistula and the level of bowel obstruction. U/S is useful in detecting the presence of cholelithiasis, choledocholithiasis and impacted stones. Gastroduodenoscopy is useful in detecting a stone impacted in duodenum. CT with gastrographin is useful in detecting a fistula, impacted stones, and pneumobilia. The reported accuracy of preoperative diagnosis ranges between $13 \%$ - 48\% [13]. In our series only four patients (50\%) received a correct preoperative diagnosis. Contrast $\mathrm{x}$-ray films showed aerobilia and dystope radiopaque stone in 2 patients and sings of obstruction in 7 patients. In three patients CT findings showed aerobilia, the fistula and an intraluminal abnormality in the terminal ileum. In most series the operative diagnosis increased to $74 \%$. In our series was $100 \%$. All patients were operated on 2 - 3 days after admission.

A gallstone must be at least $2-2.5 \mathrm{~cm}$ in diameter to cause intestinal obstruction in the normal bowel. Gallstones smaller than $2.5 \mathrm{~cm}$ usually pass spontaneously. Almost always the stone enters the gastrointestinal tract through a biliary-enteric fistula, usually of the cholecystoduodenal type. Gallstones commonly impact in the terminal ileum and the ileocecal valve, the narrowest parts of the small bowel where the peristalsis is weaker than at more proximal parts [14]. In the present report (7 pts) $87.5 \%$ of the stones were impacted in the terminal ileum and $12.5 \%$ (1 pts) impacted in duodenum (syndrome Bouveret's) (Figures 4 and 5) .The size of obstructing stones was in the range of $3-5.5 \mathrm{~cm}$.

Treatment involves an exploratory laparotomy not only to remove the stone causing the obstruction, but to carefully inspect the remainder of the small intestine for additional calculi [1]. In our series removal of the stone via enterotomy was the technique of choice in 7 patients. We performed a longitudinal incision on the antimesenteric border proximal to the site of impaction and then we closed the gut transversely to avoid narrowing of the intestinal lumen. Some authors emphasize that fistula repair and cholecystectomy should also be undertaken whenever possible, either during the initial procedure 


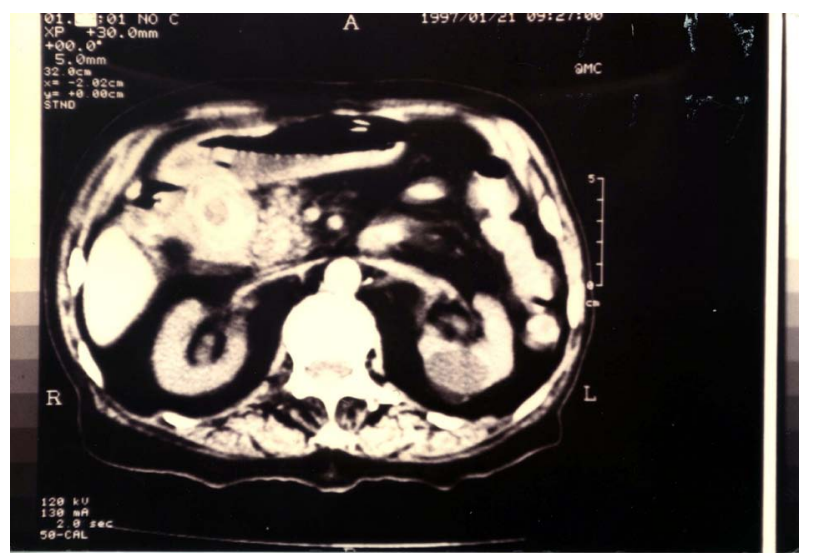

Figure 4. Upper abdominal CT image showing impacted gallstone in the duodenum.

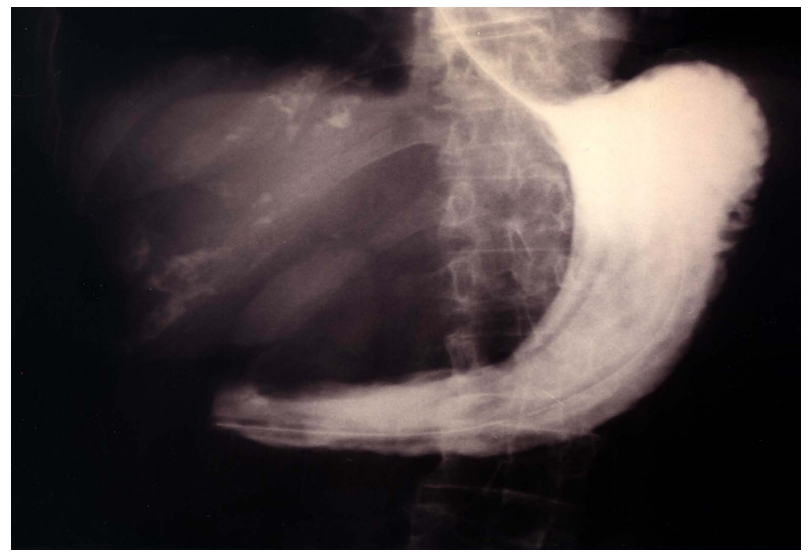

Figure 5. Contrast abdominal x-ray showing duodenal obstruction.

(one stage procedure) or at a second stage operation [15]. They argue in the removal of the gallbladder at the point of prevention further symptoms such as: recurrent gallstone ileus, cholangitis and carcinoma of the gallbladder. According to the literature $5 \%$ of patients who undergo enterolithotomy alone go on to develop biliary symptoms and $10 \%$ require an unplanned reoperation. Acute cholangitis following gallstone ileus should only occur in circumstances when the fistula or cystic duct is no longer patent and there is obstruction within the common bile duct.

There is also no comparison between the incidence of gallbladder cancer in patients undergoing cholecystectomy and those with biliary enteric fistula [16]. One patient in our series had concomitant carcinoma of the gallbladder. This patient underwent one stage procedure.

The carcinoma could have an etiological role in the formation of the fistula. In our series we had one death due to lung emboli (12.5\% mortality). Reisner et al. reviewed a series of 1001 reported cases concluded that the one stage procedure carries an associated mortality of $16.9 \%$, compared to $11.7 \%$ for simple enterolithotomy.
Also he suggested that morbidity after enterolithotomy is low [7]. Historically, wound infections and dehiscence have been cited as being the most common complications after surgery in $25 \%$ to $50 \%$ of GI cases. In our series median hospital stay was 22 days and postoperative complications occurred in 3 out of 8 patients. In one patient wound infection occurred in spite of systemic antibiotic prophylaxis and in other two patients complications were related to the type of concomitant disorders (one patient DVT-one patient atelectasia). Bibliography shows that gastrointestinal complications related to anastomotic leaks and intrabdominal abcesses are highest in patients undergoing enterotomy with fistula closure.

\section{Conclusion}

Gallstone ileus is perhaps the most insidious and difficult to diagnose cause of mechanical intestinal obstruction. It is often misdiagnosed and carries a significant rate of mortality and morbidity. Its danger reflects the advanced age of patients and the high incidence of severe concomitant diseases. Surgical treatment mandates removal of the stone via enterotomy. Whether to perform a cholecystectomy at the time of laparotomy remains controversial.

\section{Acknowledgements}

We would like to thank the consultants of the Department of Surgery, Sismanoglion general hospital Athens, for kindly allowing their patients to be included in the series.

\section{REFERENCES}

[1] X.-Z. Dai, G.-Q. Li, F. Zhang, X.-H. Wang and C.-Y. Zhang, "Gallstone Ileus: Case Report and Literature Review,” World Journal of Gastroenterology, Vol. 19, No. 33, 2013, pp. 5586-5589.

[2] J. Norton, R. Bollinger, A. Chang, S. Lowry, S. Mulvihill, H. Pass and R. Thomposon, "Surgery-Basic Science and Clinical Evidence,” Springer, New York, 2009.

[3] R. Hermosa, C. Cazador, G. Vila, R. Garo, F. Francesch and A. Fernandez, "Gallstone Ileus: Results of Analysis of a Series of 40 Patients," Gastroenterology and Hepatology, Vol. 24, No. 10, 2001, pp. 489-494.

[4] W. Hempfling, C. Rust, M. Sackmann, G. U. Muller, H. Arbogast and B. Goke, "Case Report," Medizinische Klinik, Vol. 96, No. 12, 2001, pp. 735-739. http://dx.doi.org/10.1007/PL00002170

[5] F. Scarpa, J. Borges and D. Mullen, "Clinical Image," The American Journal of Surgery, Vol. 180, 2000, p. 99.

[6] R. Grassi, A. Pinto, E. Rossi, G. Rossi, M. Scaglione and F. Lassandro, "9 Consecutive Patients with Gallstone Ileus. Personal Experience,” Radiologia Medica (Torino), Vol. 95, No. 3, 1998, pp. 177-181.

[7] R. M. Reisner and J. R. Cohen, "Gallstone Ileus: A Re- 
view of 1001 Reported Cases,” The American Surgeon, Vol. 60, No. 6, 1994, pp. 441-446.

[8] J. Hildebrandt, U. Herrmann and H. Diettrich, "Gallstone Ileus. A Report of 104 Cases,” Chirurgic, Vol. 61, No. 5, 1990, pp. 392-395.

[9] H. P. Grewal, N. J. Everitt and D. P. Fossard, "Gallstone ileus,” British Journal of Surgery, Vol. 78, No. 3, 1991, p. 378.

[10] P. A. Clavien, J. Richon, S. Burgan and A. Rohner, “Gallstone Ileus,” British Journal of Surgery, Vol. 77, No. 7, 1990, pp. 737-742. http://dx.doi.org/10.1002/bjs.1800770707

[11] L. G. Rigler, C. N. Borman and J. F. Noble, "Gallstone Obstruction: Pathogenesis and Roentgen Manifestations," The Journal of the American Medical Association, Vol. 117, No. 21, 1941, pp. 1753-1759. http://dx.doi.org/10.1001/jama.1941.02820470001001

[12] E. J. Balthazar and L. S. Chechter, "Air in Gallbladder: A
Frequent Finding in Gallstone Ileus,” American Journal of Roentgenology, Vol. 131, No. 2, 1978, pp. 219-222.

[13] Y. Kasahara, H. Umemura, S. Shiraha, T. Kuyama, K. Sakata and H. Kubota, „Gallstone Ileus: Review of 112 Patients in the Japanese Literature," The American Journal of Surgery, Vol. 140, No. 3, 1980, pp. 437-440. http://dx.doi.org/10.1016/0002-9610(80)90185-3

[14] O. H. Piedad and P. B. Wels, "Spontaneous Internal Biliary Fistula, Obstructive and Non-Obstructive Types," Annals of Surgery, Vol. 175, No. 1, 1972, pp. 75-80. http://dx.doi.org/10.1097/00000658-197201000-00013

[15] S. D. Berliner and L. C. Burson, "One-Stage Repair for Cholecystoduodenal Fistula and Gallstone Ileus," Archives of Surgery, Vol. 90, No. 2, 1965, pp. 313-316. http://dx.doi.org/10.1001/archsurg.1965.01320080137028

[16] P. A. Bossart, A. H. Patterson and H. A. Zintie, "Carcinoma of the Gallbladder," The American Journal of Surgery, Vol. 103, 1962, pp. 361-364. 